\title{
Aktuelle Therapie des Hallux valgus
}

\author{
Markus Wünschel, Nikolaus Wülker
}

\section{Zusammenfassung}

Moderne Hallux-valgus-Chirurgie erfordert ein differenziertes operatives Vorgehen anhand der zugrunde liegenden Deformität. Häufig werden Weichteileingriffe kombiniert mit Osteotomien eingesetzt zur optimalen und dauerhaften Korrektur der Fehlstellung. Bei jüngeren Patienten mit kongruentem Großzehengrundgelenk haben sich distale Osteotomien am Metatarsale bewährt. Das arthrotisch veränderte Gelenk bei älteren Patienten wird je nach Aktivitätsanspruch mit einer Resektionsarthroplastik oder einer Arthrodese therapiert.

\section{Current Treatment of Hallux valgus Deformity}

Current concepts of hallux valgus surgery require a sophisticated approach depending on the underlying deformity. A combination of soft-tissue procedures combined with osteotomies is usually necessary to achieve an optimal and lasting correction. In younger patients with a congruent metatarsophalangeal joint, distal metatarsal osteotomies are well established. The degenerative joint in older patients should be treated by resection arthroplasty or arthrodesis.

\section{Einleitung}

Obwohl der Hallux valgus schon seit Mitte des 19. Jahrhunderts operativ behandelt wird, hat sich die Therapie in den letzten Jahrzehnten weiter entwickelt. Bislang sind bereits über 150 Operationsverfahren zur Therapie des Hallux valgus bekannt, sodass wesentliche technische Neuerungen nicht zu erwarten sind [10]. Der entscheidende Fortschritt besteht vielmehr in der differenzierten Anwendung einzelner Operationsmethoden. Hallux-valgus-Deformitäten unterscheiden sich nämlich deutlich, z. B. im Zustand des Metatarsophalangealgelenkes, in der Kongruenz der Gelenkflächen und in der Ausprägung eines Metatarsus primus varus, der häufig mit dem Hallux valgus vergesellschaftet ist. Auch die Patienten unterscheiden sich im Hinblick auf ihre körperliche Aktivität, ihre Erwartungen von der Operation und im bevorzugt getragenen Schuhwerk. Nicht alle Halluxvalgus-Deformitäten können daher mit

OP-JOURNAL 2007; 23: 148-153

(C) Georg Thieme Verlag KG Stuttgart • New York nur einem Operationsverfahren befriedigend korrigiert werden. Die optimale Vorgehensweise wird vielmehr anhand von verschiedenen Kriterien ausgewählt und im Folgenden verdeutlicht.

\section{Anamnese und Untersuchungsbefund}

Hallux-valgus-Patienten klagen in erster Linie über einen druckschmerzhaften Ballen, also eine nach medial gerichtete Vorwölbung am Metatarsale-I-Kopf. Dieser Ballen ist insbesondere beim Tragen von geschlossenen Schuhen schmerzhaft. Durch die Abweichung der Großzehe nach lateral bleibt den kleinen Zehen im Schuh nicht mehr ausreichend Platz, sodass sich hier Druckbeschwerden zwischen den Zehen selbst und zum Schuh ergeben. Die gestörte Mechanik des Metatarsophalangealgelenkes der Großzehe führt zu einer Fehlbelastung und zu Schmerzen im Gelenk. Schließlich verlagert sich aufgrund der gestörten Großzehenfunktion die Belastung beim Abrollen auf die lateral gelegenen Strahlen.
Unter den Mittelfußköpfen II bis IV kommt es daher häufig zu Belastungsbeschwerden und Schwielenbildung, die als „Transfermetatarsalgie“ bezeichnet werden.

Im klinischen Befund steht die Abweichung der Großzehe nach lateral im Vordergrund. Zusätzlich ist die Großzehe häufig in Pronationsrichtung gedreht. Von Bedeutung ist eine Abweichung des Metatarsale I nach medial, die als „Metatarsus primus varus“ bezeichnet wird. Dadurch wirken die Mittelfußköpfe aufgespreizt, der Metatarsale-I-Kopf tritt noch weiter nach medial hervor (Abb.1). Dort finden sich in der Regel eine druckschmerzhafte Rötung, häufig auch eine Schwellung, gelegentlich ein in der Bursa befindlicher Erguss. In manchen Fällen kann es hier sogar zu einer Ulzeration kommen. Die kleinen Zehen treten nach oben, sodass sich hier Hammerzehen (bei denen die Zehenspitze noch den Boden berührt) oder Krallenzehen (bei denen die Zehenspitze vom

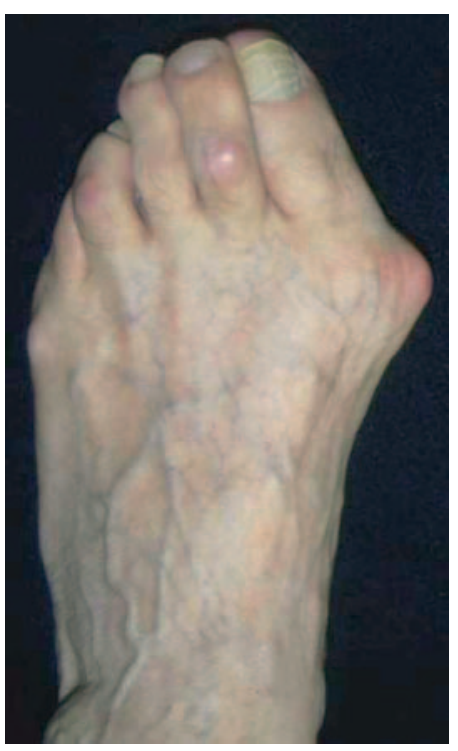

Abb. 1 Hallux valgus mit Metatarsus primus varus. 
Boden abgehoben ist) bilden. Die Plantarseite der Mittelfußköpfe II bis IV ist häufig druckschmerzhaft, oft findet sich hier auch eine Schwielenbildung als Anzeichen der Überlastung. Die Prüfung der Fußpulse sowie der Hautdurchblutung ist essenziell, um Komplikationen in der Hallux-valgus-Chirurgie zu verhüten.

\section{Röntgenbefund}

Entscheidend für die Indikationsstellung ist eine dorsoplantare Aufnahme des Vorfußes unter Belastung. Eine seitliche Aufnahme unter Belastung wird zwar routinemäßig angefordert, ist jedoch weniger aussagekräftig. Eine Aufnahme ohne Belastung kann nicht herangezogen werden, da sich das Ausmaß des Hallux valgus und des Metatarsus primus varus entscheidend mit der Belastung verändert.

Die Röntgenbilder werden zunächst auf degenerative Veränderungen am Metatarsophalangealgelenk beurteilt. Finden sich eine Gelenkspaltverschmälerung, Osteophyten oder Gelenkflächenunregelmäßigkeiten, so ist davon auszugehen, dass bereits erhebliche degenerative Veränderungen an den Gelenkflächen bestehen. In diesem Fall sind rekonstruktive Verfahren zur Korrektur des Hallux valgus nur noch eingeschränkt erfolgreich.

Als nächstes wird entschieden, ob das Großzehengrundgelenk kongruent oder inkongruent (d. h. subluxiert) ist. Letzteres ist wesentlich häufiger der Fall. Die Inkongruenz bedeutet, dass der Hallux valgus durch eine Imbalance der Weichteile um das Großzehengrundgelenk entsteht.

Zur Korrektur muss die Weichteilbalance wiederhergestellt werden. Bei geringer Inkongruenz kann man sich am Röntgenbild mithilfe von Punkten orientieren, die auf die Enden der Gelenkflächen gelegt werden. Das Gelenk ist inkongruent, wenn diese Punkte nicht aufeinander treffen.

Beim inkongruenten Grundgelenk findet sich häufig gleichzeitig eine Abweichung des Metatarsale I nach medial (Metatarsus primus varus). Sie wird auf der belasteten Aufnahme mit dem Intermetatarsalwinkel bestimmt, der zwischen den Schaftachsen des Metatarsale I und II gemessen wird (Normalwert $<10^{\circ}$ ). Bei einem vergrößerten Winkel muss die Stellung des Metatarsale I ggf. ebenfalls operativ korrigiert werden.

Bei kongruentem Gelenk sind nicht die Weichteile für den Hallux valgus verantwortlich, sondern es besteht eine knöcherne Deformität oder der Hallux valgus ist nur gering ausgeprägt. Meistens ist die Gelenkfläche des Metatarsale-I-Kopfs vermehrt nach lateral gekippt. Der distale Gelenkflächenwinkel (DMAA) zwischen einer Senkrechten auf der Metatarsale-I-Schaftachse und einer Linie durch die Gelenkflächenenden ist dann vergrößert (Normalwert 5 bis 10 Grad).

Eine knöcherne Deformität kann auch an der Grundphalanx bestehen. In diesem Fall ist der Winkel zwischen der proximalen Gelenkfläche der Grundphalanx und ihrer Schaftachse vergrößert (Normalwert 5 bis 10 Grad). Dies wird als Hallux valgus interphalangeus bezeichnet.

Begleiterscheinungen des Hallux valgus, z.B. Kleinzehendeformitäten oder eine Metatarsalgie, werden nicht anhand von Röntgenaufnahmen, sondern klinisch diagnostiziert. Eine weitere bildgebende Diagnostik, etwa mittels Schnittbilddiagnostik ist in der Regel nicht erforderlich.

\section{Konservative Therapie}

Schienen und Orthesen zur Korrektur des Hallux valgus stehen in unterschiedlichen Modellen zur Verfügung. Sie sind jedoch nicht geeignet, den Hallux valgus dauerhaft zu korrigieren. Mit ihnen kann allenfalls am wachsenden Skelett eine Progredienz der Deformität verhütet werden.

Die konservative Behandlung richtet sich in erster Linie auf die Begleitsymptome des Hallux valgus. Am erfolgreichsten ist sie bei der Metatarsalgie, wo Einlagen mit retrokapitaler Pelotte und Vorfußweichbettung meist eine deutliche Beschwerdelinderung erzielen. Druckstellen zwischen den Zehen oder gegen den Schuh können mit konfektionierten Polstern gut entlastet werden. An den Kleinzehen ist jedoch mit Richtern oder Zügeln keine dauerhafte Stellungskorrektur zu erzielen. Polsterungen an der Innenseite des Ballens sind weniger hilfreich, da sie zusätzlich im Schuh auftragen und den Druck auf die Haut noch erhöhen. Lokale oder systemische Antiphlogistika werden nur in
Ausnahmefällen, z. B. beim akuten Reizzustand eines Ballens, verordnet. Redressierende Bewegungsübungen an den Zehen sind sinnvoll, um Kontrakturen $\mathrm{zu}$ verhindern. Sie können in aller Regel vom Patienten selbst durchgeführt werden.

Zur konservativen Therapie gehört auch eine Beratung des Patienten über geeignetes Schuhwerk. Schuhe mit zu engem und spitz zulaufendem Vorfußbereich führen zwangsläufig zur Ausbiegung der Großzehe nach lateral und verstärken die Beschwerden über dem Ballen. Bei einem übermäßig hohen Absatz wird der Fuß bei jedem Schritt in den vorderen Schuhbereich gedrückt und die Zehen werden komprimiert. Eine dünne Ledersohle federt beim Auftritt nur unzureichend und verstärkt Beschwerden im Sinne einer Metatarsalgie.

\section{Operative Therapie}

Bestehen beim Hallux valgus ausreichende Beschwerden und wünscht der Patient eine Korrektur, ist eine Halluxvalgus-Operation indiziert. Wichtigste Kontraindikation ist eine mangelhafte Blutversorgung aufgrund einer arteriellen Verschlusskrankheit. Auch übermäßige Erwartungen des Patienten, insbesondere beim postoperativ zu tragenden Schuhwerk, sollten zum Überdenken der Indikation Anlass geben. Ein Eingriff bei beschwerdefreien Patienten allein zur kosmetischen Korrektur ist nicht indiziert.

Die Wahl des Operationsverfahrens bleibt in gewissem Maße den Erfahrungen des Operateurs überlassen. Mit der gewählten Technik müssen jedoch alle Komponenten der Hallux-valgus-Deformität korrigiert werden. Der Operateur wird daher eine Auswahl unterschiedlicher Operationsmethoden vorhalten müssen, um unterschiedlichen Deformitäten und unterschiedlichen Patienten gerecht werden zu können. Bei der Wahl der Operation leistet ein Therapieschema Hilfestellung, das jedoch nicht die Erfahrung und Abwägung des Operateurs ersetzt (Abb. 2).

Die häufigste Situation ist ein Hallux valgus mit Inkongruenz des Großzehengrundgelenks und ohne degenerative Veränderungen (Abb. 3). Meist sind dies mäßiggradige bis starke Hallux-valgusDeformitäten. 


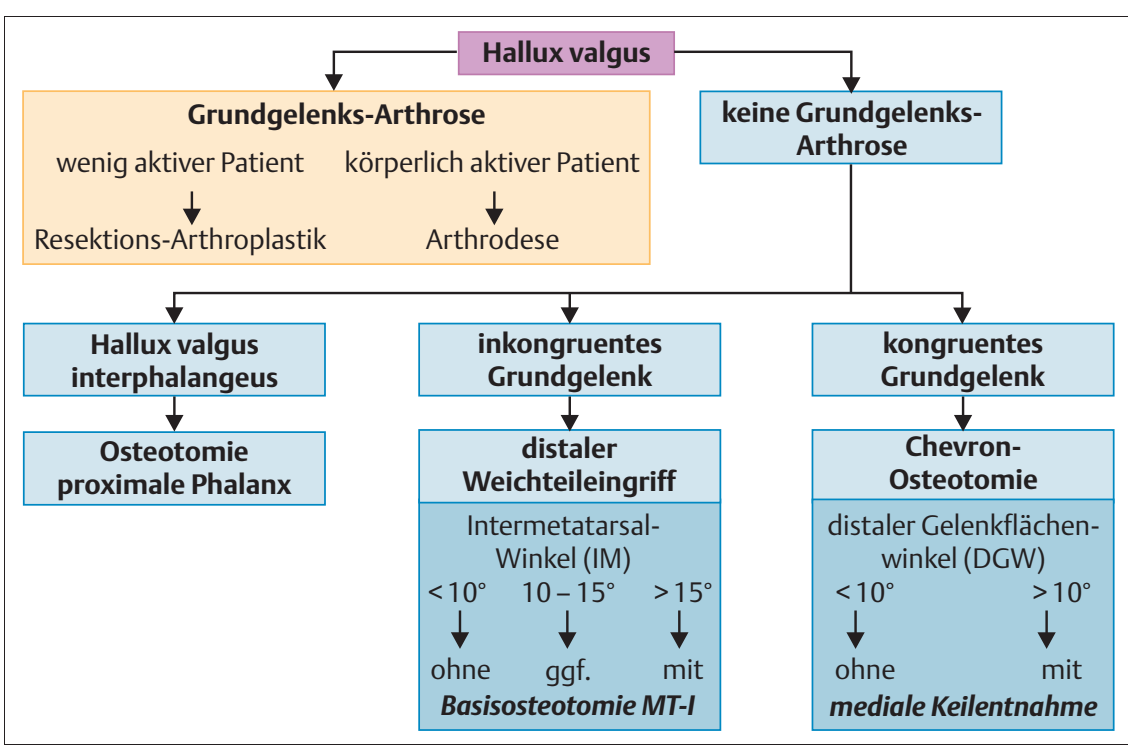

Abb. 2 Entscheidungsbaum zur Therapie des Hallux valgus.

Begleitend besteht bei der Mehrzahl der Patienten ein Metatarsus primus varus. Die Abweichung der Großzehe nach lateral kommt durch eine gestörte Weichteilbalance um das Großzehengrundgelenk zustande. Die knöcherne Anatomie und insbesondere die Ausrichtung der Gelenkflächen am ersten Strahl ist jedoch normal. Diese Form des Hallux valgus wird mit einem Weichteileingriff am Großzehengrundgelenk korrigiert, bei dem die kontrakten Weichteile auf der lateralen Seite des Gelenks entfächert und die übermäßig langen und insuffizienten Weichteile auf der Medialseite des Gelenks gerafft werden. Dieser „distale Weichteileingriff“ [7,8] wird mit einer proximalen Osteotomie des Metatarsale I kombiniert, wenn der Intermetatarsalwinkel deutlich über 10 Grad beträgt. Sie ist erforderlich, um den Metatarsus primus varus zu korrigieren. Die Osteotomie erfolgt proximal, da hier das Korrekturpotenzial am größten ist. Nur selten besteht bei diesen Patienten eine so ausgeprägte Hypermobilität des ersten Tarsometatarsalgelenkes, dass eine Basisosteotomie keine ausreichende Korrektur erzielt und stattdessen eine Arthrodese des hypermobilen Gelenks erforderlich wird.

Weniger häufig ist beim Hallux valgus ohne arthrotische Veränderungen das Großzehengrundgelenk kongruent. Meist sind dies nur leicht- bis mäßiggradige Deformitäten, oft bei jüngeren Patientinnen und Patienten. Hier kann der distale Weichteileingriff nicht verwendet werden, da das Gelenk sonst in eine inkongruente Stellung gebracht würde.
Vielmehr erfolgt eine knöcherne Korrektur am Metatarsale-I-Kopf. Dabei wird das Kopffragment nach lateral verschoben. Bei vergrößertem distalen Gelenkflächenwinkel (DMAA) wird gleichzeitig ein medialbasiger Keil entnommen, um die Gelenkfläche anatomisch zum Metatarsale-I-Schaft auszurichten. Eine große Zahl verschiedener Osteotomien steht zur Verfügung. Besonders geeignet sind Osteotomien, die in zwei Ebenen verlaufen und dadurch eine besondere Stabilität gewährleisten. Hier hat sich insbesondere die Chevron-Osteotomie bewährt [2].

Ein Hallux valgus interphalangeus wird selten für sich alleine durch eine Osteotomie der Grundphalanx mit Entnahme eines medialbasigen Keils korrigiert [1]. Eine Indikation zu dieser Osteotomie ergibt sich jedoch gelegentlich, wenn die Großzehe nach Abschluss des distalen Weichteileingriffs bei ausgerichtetem Großzehengrundgelenk noch deutlich nach lateral abweicht.

Bei Patienten mit degenerativen Veränderungen am Großzehengrundgelenk sind die genannten rekonstruktiven Operationstechniken nicht Erfolg versprechend (Abb. 4).

Bei diesen Patienten liefert die Resektionsarthroplastik [3,5] durch Debasierung der Grundphalanx nach wie vor die besten Ergebnisse. Degenerative Veränderungen am Großzehengrundgelenk in einem Alter unter 60 Jahren sind selten. Diese Patienten werden vorzugsweise mit einer Arthrodese des

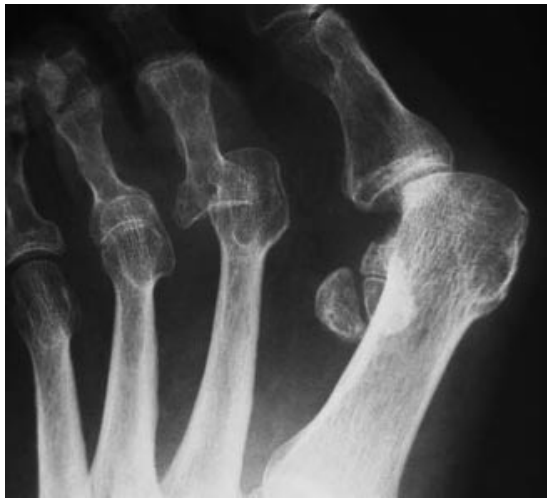

Abb. 3 Deutlich inkongruentes Großzehengrundgelenk.

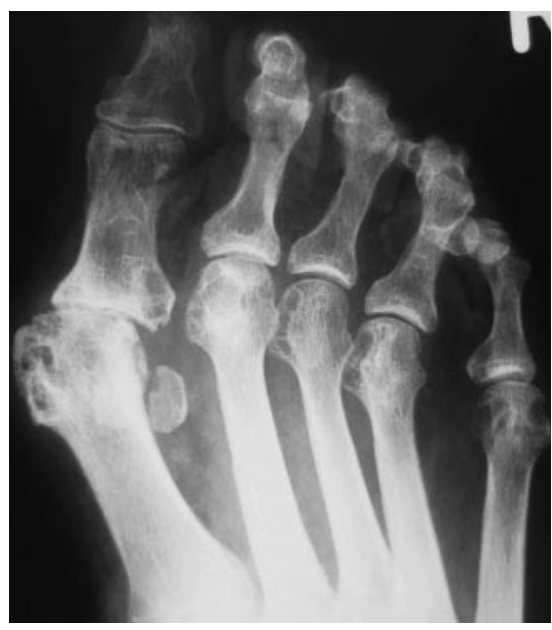

Abb. 4 Hallux valgus mit starken degenerativen Veränderungen.

Großzehengrundgelenks versorgt, da diese die Funktion der Großzehe beim Abstoßen während des Gehens wesentlich besser erhält als die Resektionsarthroplastik. In gleicher Weise wird bei einem Hallux-valgus-Winkel von über 50 Grad verfahren, da hier Rekonstruktionen nicht mehr Erfolg versprechend sind [4].

Gleichzeitige Korrekturoperationen an den kleinen Zehen sind nur erforderlich, wenn hier Deformitäten vorliegen. Eine Metatarsalgie bessert sich in der Regel alleine durch die Großzehenkorrektur ausreichend, sodass Metatarsale-Osteotomien (z.B. die Weil-Osteotomie) zunächst nicht durchgeführt werden.

Wichtigste Kontraindikation zu Eingriffen bei Hallux valgus sind Durchblutungsstörungen. Weitere diagnostische Maßnahmen sind erforderlich, wenn nicht beide Fußpulse deutlich zu tasten sind. 


\section{Operationstechniken}

Hallux-valgus-Operationen können in regionaler, spinaler oder allgemeiner Anästhesie durchgeführt werden. Die meisten Eingriffe können ambulant vorgenommen werden. Die Operationen erfolgen in der Regel in Blutleere, welche in Esmarch-Technik angelegt wird. Die Hautfäden sollen am Fuß 14 Tage belassen werden. Die Redression der Großzehe durch einen Verband in den Wochen nach der Operation trägt wesentlich zum Erfolg bei.

\section{Distaler Weichteileingriff}

Zu Beginn erfolgt das laterale Release am Großzehengrundgelenk durch einen dorsalen Hautschnitt im ersten Intermetatarsalraum.

Nach Eingehen direkt bis auf die Adduktorensehne und Darstellen mit Haken wird die Sehne mit dem Skalpell von proximal nach distal von der lateralen Seite des fibularen Sesambeins und von der Grundphalanx abgelöst. Direkt darunter befindet sich das Ligamentum metatarseum transversum.

Dieses Band wird zur Schonung der direkt darunter verlaufenen Nerven und Gefäße zunächst mit einer gebogenen Klemme unterfahren und anschließend durchtrennt (Abb.5). Die laterale Gelenkkapsel wird anschließend mit einem Skalpell mehrfach gestichelt, bis die Großzehe in eine Varusposition von 30 Grad gebracht werden kann, wobei die Kapsel häufig hörbar und fühlbar einreißt.

Der zweite Hautschnitt erfolgt direkt medial über der Pseudoexostose. Das meist verdickte Kapsel- und Bursagewebe über der Pseudoexostose wird L-förmig inzidiert, wobei der vertikale Schenkel etwas proximal zum Gelenkspalt liegt. Die Pseudoexostose wird dargestellt und in Verlängerung der medialen Metatarsale-I-Diaphyse mit dem Meißel oder der Säge abgetragen (Abb. 6).

Ein Metatarsus primus varus muss gleichzeitig mit dem Weichteileingriff korrigiert werden. Bei einem Intermetatarsalwinkel zwischen 10 und $15^{\circ}$ wird geprüft, ob das Metatarsale I ohne wesentliche, federnde Gegenkraft an das Metatarsale II angenähert werden kann. In diesem Fall reichen kräftige, resorbierbare Nähte aus, die in den ersten Intermetatarsalraum in den proximalen

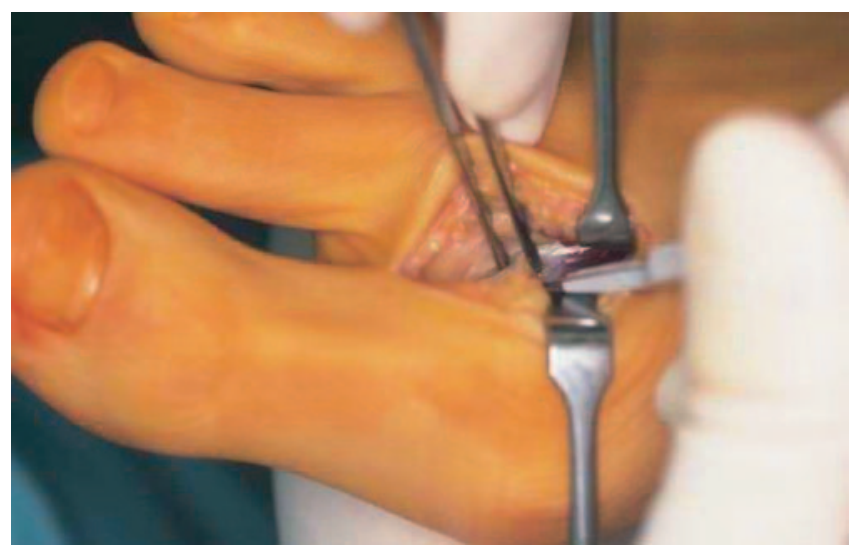

Abb. 5 Das Ligamentum metatarseum transversum wird durchtrennt.

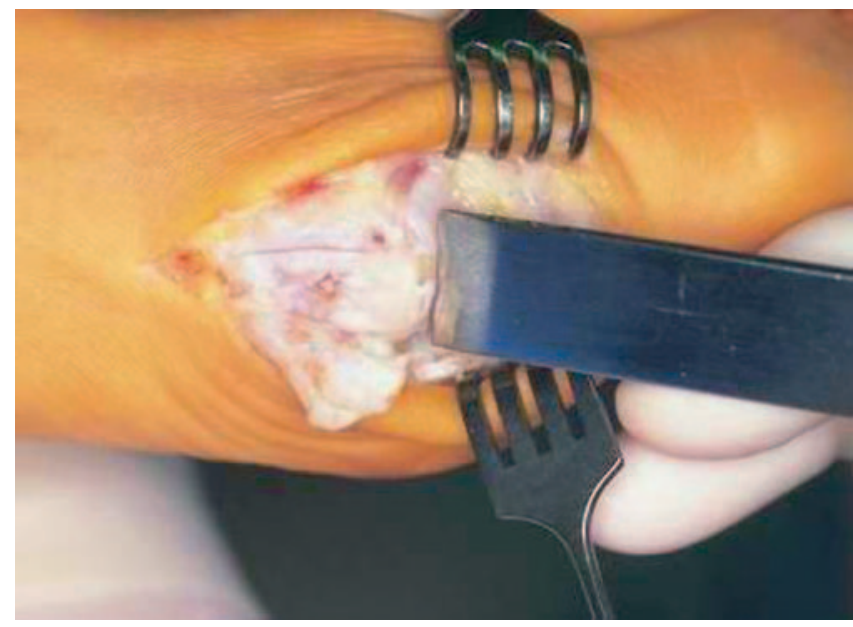

Abb. 6 Abtragung der Pseudoexostose.

Anteil der Gelenkkapseln I und II gelegt werden.

Eine Basisosteotomie am Metatarsale I ist bei einem Intermetatarsalwinkel von über 15 Grad und, wenn die Stellung des Metatarsale I bei geringeren Winkeln nicht ohne Widerstand korrigiert werden kann, obligat [6].

Die Basisosteotomie erfolgt vorzugsweise durch einen dritten Hautschnitt, der proximal und zwischen die beiden ersten Schnitte gelegt wird. Verschiedene Osteotomietechniken können verwendet werden. Wir bevorzugen eine nach distal geöffnete Chevron-Osteotomie, deren dorsaler Schenkel nahezu vertikal und knapp $1 \mathrm{~cm}$ distal zum ersten Tarsometatarsalgelenk liegt und deren plantarer Schenkel in einem Winkel von etwa 135 Grad nach distal und plantar reicht. Der dorsale Schenkel der Osteotomie wird um den gewünschten Korrekturwinkel geöffnet. Medial kann von der Pseudoexostose entnommener Knochen eingelagert werden. Die Stabilisierung erfolgt mit einer von dorsal/distal nach proximal/plantar eingebrachten Spongiosaschraube.

Nun wird die große Zehe achsengerecht ausgerichtet und ein Streifen von 4 bis $8 \mathrm{~mm}$ Breite von der vertikalen Kapsel entnommen, sodass durch die Kapselraffung mit kräftigen resorbierbaren Fäden die Großzehe in einer leicht überkorrigierten Stellung verbleibt.

Die Patienten können bereits am ersten postoperativen Tag unter voller Belastung im Verbandsschuh mit starrer Sohle mobilisiert werden, der bis 6 Wochen postoperativ verbleibt. Gleichzeitig muss die Großzehe redressierend bandagiert werden (Abb.7). Im Anschluss erfolgen Bewegungsübungen an der Großzehe und Gehschulung in bequemem Schuhwerk.

\section{Chevron-Osteotomie}

Durch einen medialen Zugang über der Pseudoexostose wird das verdickte Kapsel- und Bursagewebe dargestellt und L-förmig inzidiert, wobei der vertikale 
Schenkel etwas proximal zum Gelenkspalt liegt. Die Pseudoexostose wird mit der Säge abgetragen, distal nur sehr wenig, proximal in Verlängerung der medialen Metatarsale-I-Diaphyse. Ein 2,5-mm-Bohrloch wird zentral in den Metatarsale-I-Kopf und in Richtung des Metatarsale-II-Kopfs gelegt (Abb.8). Mit der oszillierenden Säge wird die dorsale Osteotomie nahezu vertikal gelegt, sodass sie etwas proximal vom Kapselansatz austritt. Die plantare Osteotomie liegt eher horizontal und mündet proximal von der Metatarsale-I-Kopf-Gelenkfläche. Bei vergrößertem distalen Gelenkflächenwinkel wird aus der dorsalen Osteotomie ein medialbasiger Keil entnommen, wobei die Basis eine Breite von $2 \mathrm{~mm}$ nicht überschreiten sollte.

Nachdem das Periost auf der Lateralseite mit einem Meißel gelockert wurde, wird das Kopffragment um ein Drittel bis höchstens die Hälfte der Schaftbreite nach lateral verschoben. Am Ende müssen beide Osteotomieflächen geschlossen sein.

Die Stabilisierung erfolgt vorzugsweise mit einer Kortikalisschraube, die von dorsomedial am proximalen Fragment in den Kopf eingeschraubt wird (Abb.9). Der Schraubenkopf muss gut versenkt werden. Die Kapsel wird unter Verkürzung im vertikalen Anteil verschlossen, wozu ggf. ein 2 bis $3 \mathrm{~mm}$ breiter Kapselstreifen exzidiert werden muss.

Die postoperative Rehabilitation unter voller Belastung im Verbandsschuh über sechs Wochen schreitet meist rasch voran. Die große Zehe muss bis sechs Wochen nach der Operation redressierend bandagiert werden.

\section{Osteotomie der Grundphalanx}

Der Eingriff, der in der Regel in Kombination mit einem distalen Weichteileingriff erfolgt [9], wird durch Verlängerung der medialen Hautinzision nach distal vorgenommen. Die Grundphalanx wird am Ort der maximalen Deformität mit Hohmann-Haken umfahren. Ein medialbasiger Keil entsprechend der Deformität wird entnommen, wobei das Periost auf der Lateralseite intakt belassen wird. Die Breite der Keilbasis liegt zwischen 2 und $3 \mathrm{~mm}$. Die Osteotomie kann mit einem kräftigen, transossär gelegten resorbierbaren Faden stabilisiert werden. Klammern oder Schrauben sind nur erforderlich, wenn die Grundphalanx gleichzeitig verkürzt wird.

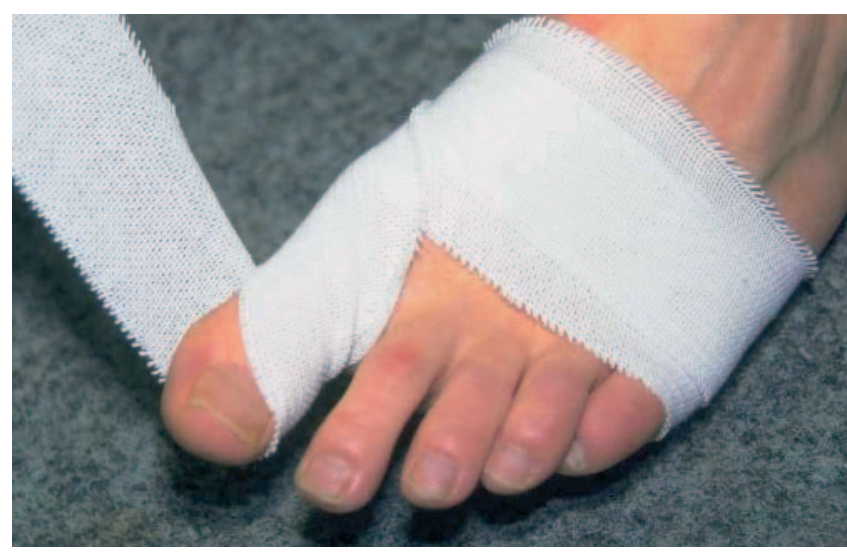

Abb. 7 Die redressierende Wickelung muss bis 6 Wochen nach der Operation fortgeführt werden.

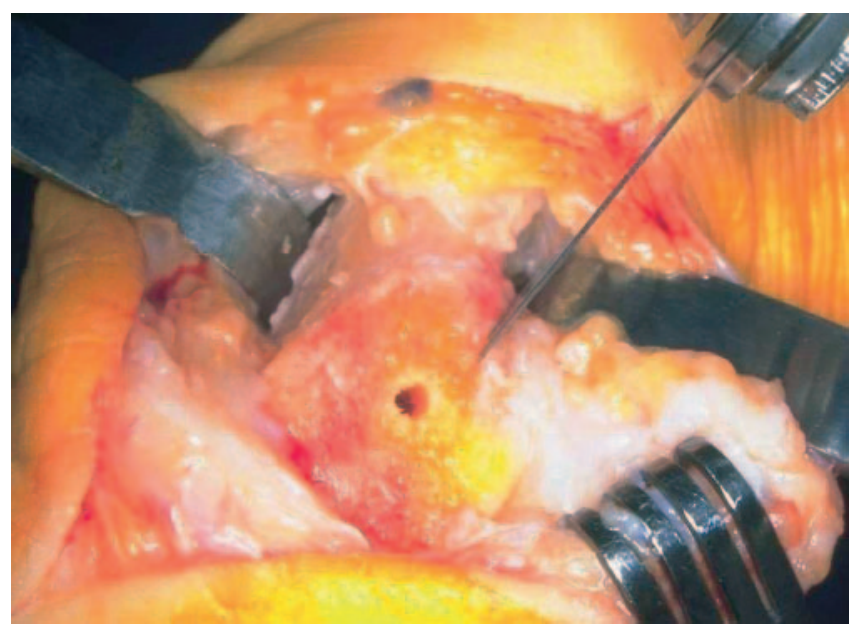

Abb. 8 Durchführung der Sägeschnitte nach Zentralbohrung.
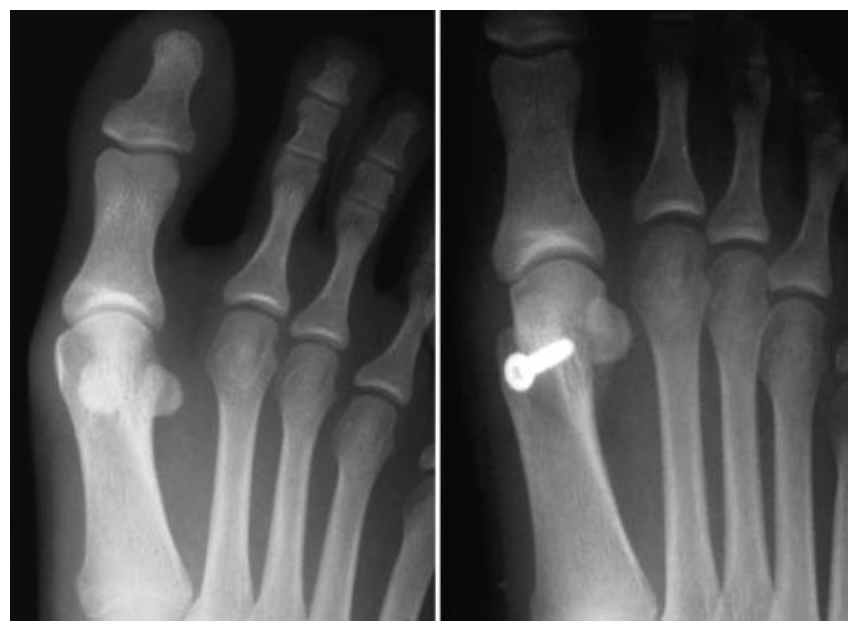

Abb. 9 Prä- und postoperatives Röntgenbild nach Chevron-Osteotomie.

\section{Resektionsarthroplastik}

Der Zugang erfolgt durch einen medialen Hautschnitt. Das verdickte Bursaund Kapselgewebe wird dargestellt und so von der Pseudoexostose abpräpariert, dass ein distal gestielter Lappen entsteht. Dabei wird auch das proximale Drittel der Grundphalanx freigelegt und mit Hohmann-Haken umfahren. Mit der
Säge wird die Pseudoexostose in Verlängerung der medialen Metatarsale-I-Diaphyse abgetragen. Anschließend wird das proximale Drittel der Grundphalanx durch eine Osteotomie senkrecht zur Schaftachse entfernt. Dabei müssen die kräftigen Insertionen der $\mathrm{Mm}$. flexor und extensor hallucis brevis durchtrennt werden. 


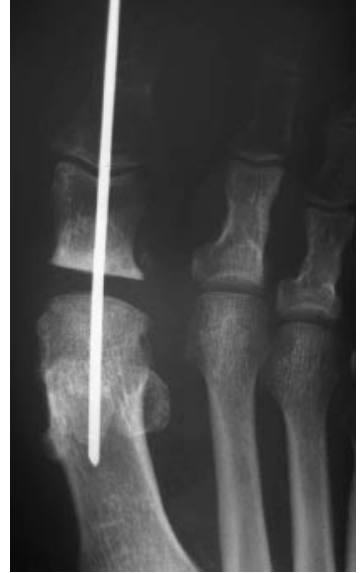

Abb.10 Röntgenbild nach Resektionsarthroplastik.

In dem etwa $1 \mathrm{~cm}$ breiten Resektionsspalt wird der Kapsellappen eingelegt und mit einem axial durch die Großzehe in das Metatarsale I eingebrachten Kirschner-Draht fixiert (Abb. 10). Bei ausgeprägtem Metatarsus primus varus kann eine zusätzlich durchgeführte Basisosteotomie vonnöten sein.

Postoperativ können die Patienten im Verbandsschuh unter voller Belastung mobilisiert werden. Der Draht wird nach zwei Wochen gezogen. Die Patienten können zu normalem Schuhwerk übergehen.

\section{Arthrodese des Großzehengrundgelenks}

Durch einen dorsalen Hautschnitt medial zur Sehne des Musculus extensor hallucis longus direkt bis auf den Knochen wird das Großzehengrundgelenk freigelegt. Osteophyten werden abgetragen. Die Gelenkfläche am Metatarsale-ISchaft wird senkrecht zur Fußsohle unter möglichst geringer Knochenresektion entfernt. Die Großzehe wird in die gewünschte Arthrodesenstellung gebracht: Sie soll die zweite Zehe gerade berühren. Die Dorsalextension beträgt

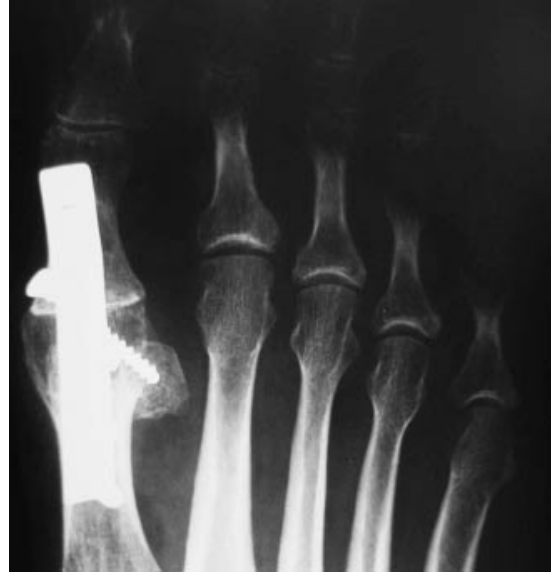

Abb. 11 Korrekte Zehenstellung durch Arthrodese.

5 bis 15 Grad zur Fußsohlenebene, 15 bis 25 Grad zum Metatarsale I. In dieser Stellung wird die Gelenkfläche der Grundphalanx wiederum unter minimaler Knochenresektion parallel zur ersten Osteotomie reseziert.

Die Arthrodese wird mit einer dorsalen Drittelrohrplatte stabilisiert, allerdings nur ohne bestehende Vorschädigung an den Weichteilen. Ansonsten werden gekreuzte Zugschrauben verwendet.

Die Drittelrohrplatte muss nur um wenige Grad gebogen werden. Zusätzlich wird eine Zugschraube, vorzugsweise von distal/medial nach proximal/lateral eingebracht (Abb.11). Bei Verwendung einer Metallplatte soll der Hautverschluss zweischichtig erfolgen, um die Platte vollständig zu bedecken.

Postoperativ können die Patienten in einem Verbandsschuh voll belasten, wenn eine Platte verwendet wurde. Ansonsten erfolgt Entlastung im Verbandsschuh bis sechs Wochen nach der Operation. Anschließend Abnahme des Verbandsschuhs und Übungsbehandlungen.

\section{Schlussfolgerung}

Die dauerhafte Korrektur einer Halluxvalgus-Fehlstellung ist durch konservative Maßnahmen nicht möglich. Nur durch die differenzierte Anwendung und teilweise Kombination verschiedener Operationstechniken ist ein langwährender Therapieerfolg zu erreichen.

\section{Literatur}

1 Akin OF. The treatment of hallux valgus: a new operative procedure and its results. Med Sentinel 1925; 33: 678-679

2 Austin DW, Leventen EO. A new osteotomy for hallux valgus. Clin Orthop 1981; 157: $25-30$

3 Brandes M. Zur operativen Therapie des Hallux valgus. Zentralbl Chir 1929; 56: 24-34

${ }^{4}$ Coughlin MJ, Grebing BR, Jones CP. Arthrodesis of the first metatarsophalan-geal joint for idiopathic hallux valgus: intermediate results. Foot Ankle Int 2005; 10: 783 - 792

${ }^{5}$ Keller WL. The surgical treatment of bunions and hallux valgus. NY Med J 1904; 80: 741

${ }^{6}$ Mann RA, Coughlin MJ. Adult hallux valgus. In: Mann RA, Coughlin MJ (Eds). Surgery of the Foot and Ankle. St. Louis: Mosby, 1992: $167-296$

7 Mann RA, Pfeffinger L. Hallux valgus repair: DuVries modified McBride procedure. Clin Orthop 1991; 272: $213-218$

8 McBride ED. A conservative operation for bunions. J Bone Joint Surg 1928; 10: 735

${ }^{9}$ Mitchell LA, Baxter DE. A Chevron-Akin double osteotomy for correction of hallux valgus. Foot Ankle Int 1991; 12: 7-14

10 Wülker N. Hallux valgus - Hallux rigidus. Stuttgart: Enke, 1997

\section{Dr. med. Markus Wünschel}

Oberarzt

Prof. Dr. med. Nikolaus Wülker

Ärztlicher Direktor

Orthopädische Klinik der Eberhard-Karls-Universität Tübingen Hoppe-Seyler-Straße 3 72076 Tübingen 